\title{
A Contourlet-Based Image Watermarking Scheme with High Resistance to Removal and Geometrical Attacks
}

\author{
Sirvan Khalighi, ${ }^{1,2}$ Parisa Tirdad, ${ }^{1}$ and Hamid R. Rabiee ${ }^{2}$ \\ ${ }^{1}$ Electical and Computer Engineering Department, Islamic Azad University of Qazvin, Iran \\ ${ }^{2}$ AICTC Research Center, Department of Computer Engineering, Sharif University of Technology, Iran
}

Correspondence should be addressed to Sirvan Khalighi, khalighi@ce.sharif.edu

Received 16 August 2009; Revised 8 January 2010; Accepted 1 June 2010

Academic Editor: Yingzi Du

Copyright (C) 2010 Sirvan Khalighi et al. This is an open access article distributed under the Creative Commons Attribution License, which permits unrestricted use, distribution, and reproduction in any medium, provided the original work is properly cited.

\begin{abstract}
We propose a new nonblind multiresolution watermarking method for still images based on the contourlet transform (CT). In our approach, the watermark is a grayscale image which is embedded into the highest frequency subband of the host image in its contourlet domain. We demonstrate that in comparison to other methods, this method enables us to embed more amounts of data into the directional subbands of the host image without degrading its perceptibility. The experimental results show robustness against several common watermarking attacks such as compression, adding noise, filtering, and geometrical transformations. Since the proposed approach can embed considerable payload, while providing good perceptual transparency and resistance to many attacks, it is a suitable algorithm for fingerprinting applications.
\end{abstract}

\section{Introduction}

Recent rapid growth of distributed networks such as Internet enables the users and content providers to access, manipulate, and distribute digital contents in high volumes. In this situation, there is a strong need for techniques to protect the copyright of the original data to prevent its unauthorized duplication. One approach to address this problem involves adding an invisible structure to a host media to prove its copyright ownership. These structures are known as digital watermarks. Digital watermarking is performed upon various types of digital contents such as images, audio, text, video, and 3D models. It is applied to many applications, such as copyright protection, data authentication, fingerprinting, and data hiding [1]. Current methods of watermarking images, depending on whether the original image is used during watermark extraction process or not, could be divided into two categories: blind and non-blind methods. Schemes reported in $[2,3]$ are nonblind methods, while the methods in [4-9] are categorized as blind methods. Most of the reported schemes use an additive watermark to the image in the spatial domain or in frequency domain. Recent works on digital watermarking for still images are applied on frequency domain.

Among the transform domain techniques, discrete wavelet transform-(DWT-) based techniques are more popular, since DWT has a number of advantages over other transforms including space-frequency localization, multiresolution representation, superior HVS modeling, linear complexity, and adaptivity [10]. In general, the DWT algorithms try to locate regions of high frequency or middle frequency to embed information, imperceptibly [11]. Even though DWT is popular, powerful, and familiar among watermarking techniques, it has its own limitations in capturing the directional information such as smooth contours and the directional edges of the image. This problem is addressed by contourlet transform (CT) [12]. The contourlet transform was developed as an improvement over wavelet where the directional information is important. In addition to multiscale and time-frequency localization proprieties of wavelets, CT offers directionality and anisotropy.

Zaboli and Moin [2] used the human visual System characteristics and an entropy-based approach to create an 
efficient watermarking scheme. It decomposes the original image in CT domain in four hierarchical levels and watermarks it with a binary logo image which is scrambled through a well-known PN sequence. They showed adding a scrambled watermark to high-pass coefficients in an adaptive way based on entropy results in a high performance detection capability for watermark extraction.

Jayalakshmi et al. [3] proposed a non-blind watermarking scheme using the pixels selected from high frequency coefficients based on directional subband which doubles at every level. They noted that contourlet-based methods perform much better than wavelet-based methods in images like maps. The watermark was a $16 \times 16$ binary logo.

Duan et al. [4] proposed a watermarking algorithm using nonredundant contourlet transform that exploits the energy relations between parent and children coefficients. This special relationship provides energy invariance before and after the JPEG compression. They embedded a pseudorandom binary watermark exploiting the modulation of the energy relations.

Xiao et al. [5] proposed an adaptive watermarking scheme based on texture and luminance features in the CT domain, which uses the texture and luminance features of the host image to find the positions in which the watermark is embedded. Salahi et al. [6] presented a new blind spread spectrum method in contourlet domain, where the watermark is embedded through a PN sequence in the selected contourlet coefficients of the cover image, and the data embedding is performed in selected subbands providing higher resiliency through better spread of spectrum compared to the other subbands.

Shu et al. [7] proposed a blind HVS-based watermarking algorithm in the translation invariant circular symmetric contourlet transform. This approach shows good resistance against Gaussian white noise attack. Lian et al. [8] presented a method based on nonsampled contourlet transform (NSCT). The algorithm provides an HVS model in the NSCT domain, exploiting the masking characteristics of the HVS to embed the watermark adaptively. Wei et al. [9] presented an adaptive watermarking method in the CT domain based on clustering of the mean shift texture features. During clustering, three texture features including energy, entropy, and contrast are selected for mean shift fast clustering. The watermark is directly embedded in the strong texture region of the host image.

In [13], we proposed a new contourlet-based image watermarking method which embeds a grayscale watermark with as much as $25 \%$ of the host image size in the 16th directional subband of the host image. Since the original image is required for watermark extraction, our method is considered to be nonblind. In this paper, we employ the method introduced in [13] with more details and some improvement in our algorithm and provide comprehensive experiments with more host images. The remainder of the paper is organized as follows. In Section 2, we present Contourlet Transform (CT). In Section 3, we introduce the proposed approach. Experimental results are discussed in Section 4. Final remarks are outlined in Section 5.

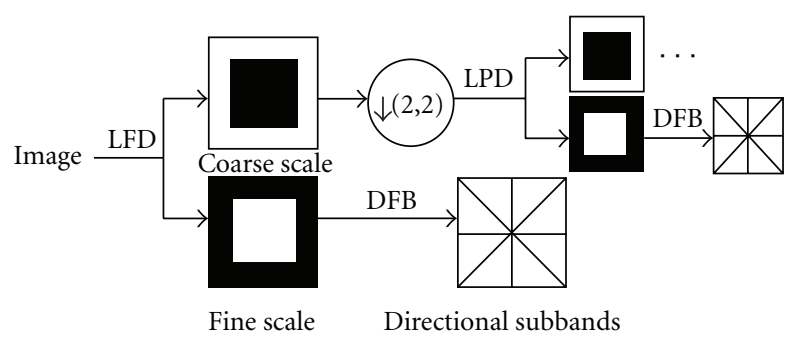

Figure 1: Contourlet filter bank [6].

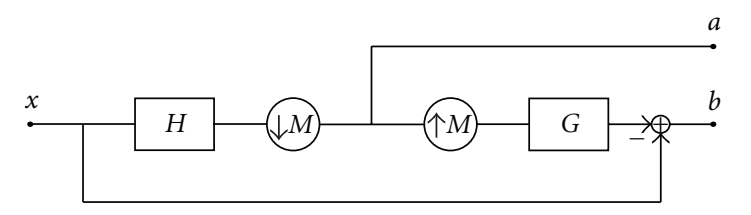

(a)

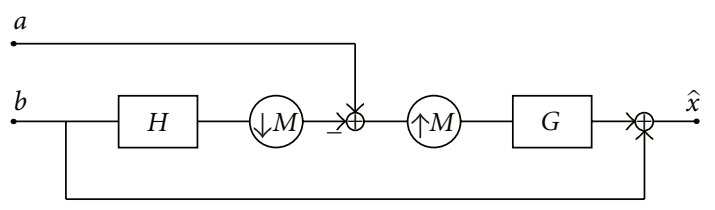

(b)

FIGURE 2: Laplacian pyramid scheme (a) analysis and (b) reconstruction [12].

\section{Discrete Contourlet Transform}

The contourlet transform (CT) is a geometrical imagebased transform that was introduced in [12]. In contourlet transform, the laplacian pyramid (LP) is first used to capture point discontinuities. It is then followed by a directional filter bank (DFB) to link point discontinuities into linear structures [14]. As shown in Figure 1, the first stage is LP decomposition and the second stage is DFB decomposition. The overall result is an image expansion using basic elements like contour segments, and thus called contourlet transform, which is implemented by a pyramidal directional filter bank (PDFB) [15]. At each level, the LP decomposition generates a downsampled lowpass version of the original, and the difference between the original and the prediction results in a bandpass image. Figure 2 illustrates this process, where $H$ and $G$ are called analysis and synthesis filters, respectively, and $M$ is the sampling matrix.

The bandpass image obtained in the LP decomposition is further processed by a DFB. A DFB is designed to capture the high-frequency content like smooth contours and directional edges. The DFB is efficiently implemented via a $K$-level binary tree decomposition that leads to $2^{K}$ subbands with wedge-shaped frequency partitioning as shown in Figure 3. The contourlet decomposition is illustrated by using the Lena test image of size $512 \times 512$ and its decomposition into four levels, in Figure 4. At each successive level, the number of directional subbands is $2,4,8$, and 16 .

Embedding the watermark in high frequency components improves the perceptibility of the watermarked image. 


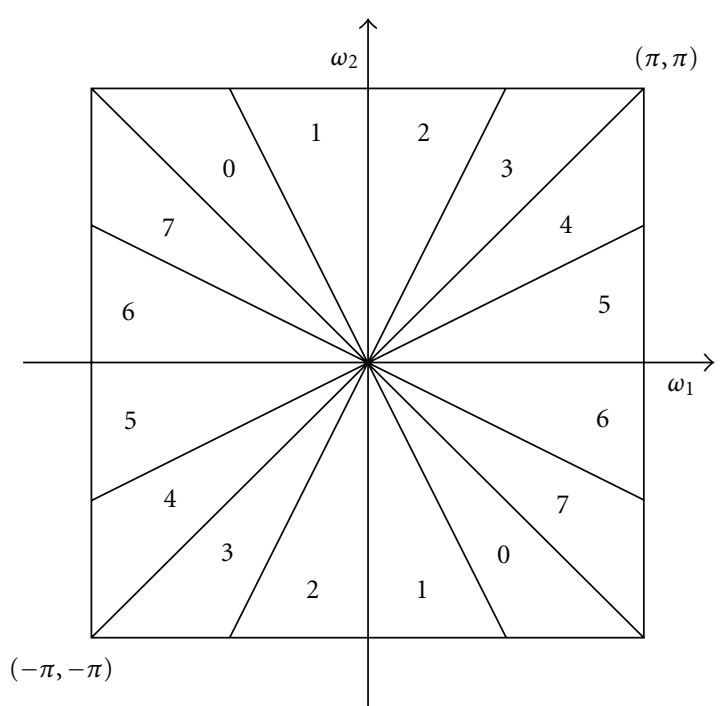

FIGURE 3: Frequency partitioning $(k=3,2 k=8$ we dge-shaped frequency subbands) [12].

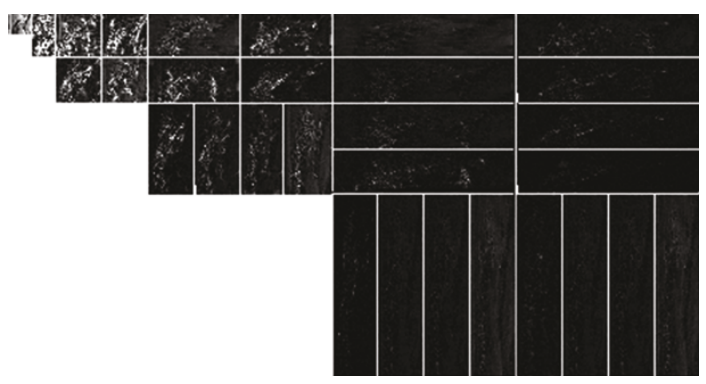

Figure 4: Contourlet decomposition of Lena.

Therefore, we have selected the highest frequency subband which possesses the maximum energy for watermark embedding (Figure 5). The Energy $E$ of a subband $s(i, j), 0 \leq i, j \leq$ $N$ is computed by

$$
E=\sum_{i} \sum_{j}|s(i, j)|^{2}
$$

The majority of coefficients in the highest frequency subband are significant values compared to the other subbands of the same level, indicating the presence of directional edges.

\section{The Proposed Approach}

We select contourlet transform for watermark embedding because it captures the directional edges and smooth contours better than other transforms. Since the human visual system is less sensitive to the edges, embedding the watermark in the directional subband improves the perceptibility of the watermarked image, but it is hardly robust. To achieve robustness, we can embed the watermark in the lowpass image of the contourlet decomposition. However, the perceptibility of the watermarked image degrades. In

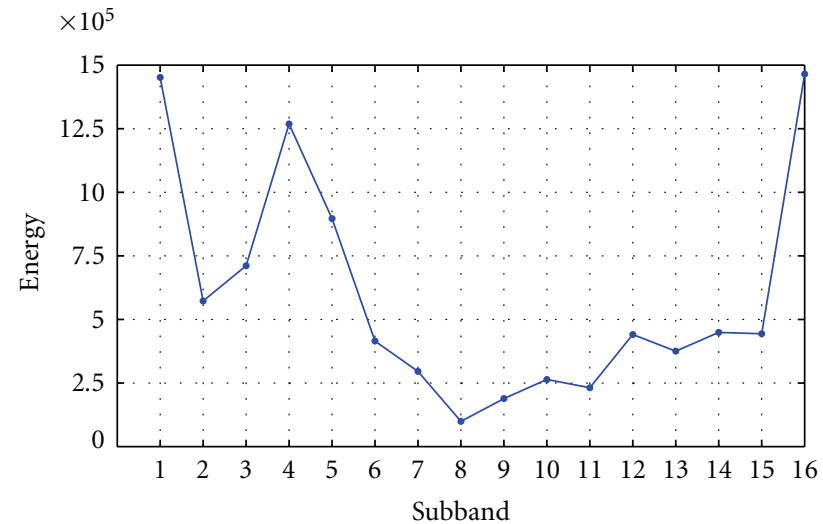

FIGURE 5: Energy variation in the last level.

our scheme, although the watermark is embedded into the highest frequency subbands, it is likely to be spread out into all subbands when we reconstruct the watermarked image, due to the special transform structure of laplacian pyramid (LP) [16]. Because the high-frequency subbands of the watermarked image contain the watermarking components, the proposed scheme is highly robust against various low-frequency attacks, which will remove the low frequency component of the image. On the other hand, some watermarking components can be preserved at the low-frequency subbands. Thus, the scheme is expected to be also robust to the high-frequency attacks, which will destroy the high-frequency components of the image. Consequently, the proposed watermarking scheme is robust to the widely spectral attacks resulting from both the low-and highfrequency processing techniques. The proposed approach is presented in Section 3.1.

3.1. Watermark Embedding Technique. In the proposed algorithm, the watermark which is a grayscale image, with as much as $25 \%$ of the host image size, is embedded into the gray level host image of size $N \times N$. The host image and the watermark are transformed into the contourlet domain.Then, the CT coefficients of the last directional subband of the host image are modified to embed the watermark. The steps involved in watermark embedding are shown in Figure 6 . We use $f(i, j)$ to denote the host image, $f^{\prime}(i, j)$ the watermarked image, and $w(i, j)$ the watermark. The technique is comprised in three main steps as discussed below.

Step 1. The host image $f(i, j)$ of size $N \times N$ and the watermark $w(i, j)$ of size $N / 2 \times N / 2$ are transformed into the CT domain. An " $n$ " level pyramidal structure is selected for LP decomposition. At each level $l_{k}$, there are $2^{l_{k}}$ directional subbands, where $k=1,2,3, \ldots, n$. The highest frequency subband of the host image is selected for watermark embedding. Watermark decomposition results in two subbands $w_{1}$, $w_{2}$ and a lowpass image. Since $w_{1}$ and $w_{2}$ have the same resolution, therefore we choose one of them, in addition to the lowpass image for watermark embedding. 


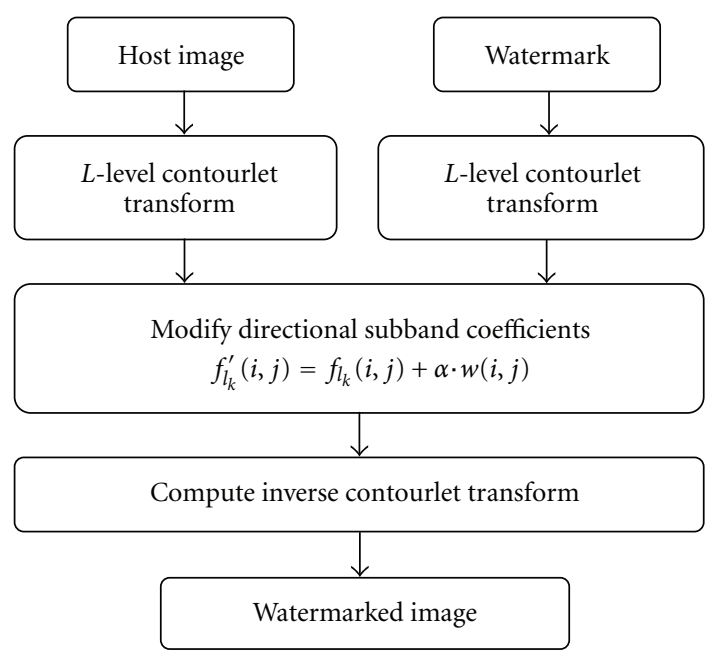

FIGURE 6: Embedding algorithm.

Step 2. The coefficients of the selected subband are modified as follows [17]:

$$
f_{l_{k}}^{\prime}(i, j)=f_{l_{k}}(i, j)+\alpha \cdot W(i, j),
$$

Where $f_{l}^{\prime}(i, j)$ represents $l$ th level, $k$ th directional subband coefficients, and $\alpha$ is a weighting factor which controls robustness and perceptual quality.

Step 3. inverse contourlet transform (ICT) is applied by considering the modified directional subbands to obtain the watermarked image.

3.2. Watermark Extraction Process. For retrieving the watermark, we need a copy of the original image as a reference. By using the inverse embedding formula (3), we can extract the embedded watermark

$$
w^{\prime}(i, j)=\frac{f_{l_{k}}^{\prime}(i, j)-f_{l_{k}}(i, j)}{\alpha} .
$$

The extraction process consists of the following steps.

Step 1. Both watermarked and original images are transformed into CT domain.

Step 2. The directional subband and the lowpass image of the embedded watermark will be retrieved by subtracting the highest frequency subbands of the original and the watermarked image by using (3).

Step 3. For reconstructing the watermark, Laplacian Pyramid requires both directional subbands $\left(\mathrm{W}_{1}, \mathrm{~W}_{2}\right)$ and the lowpass image $(\mathrm{L})$. Instead of inputting $\left(\mathrm{L}, \mathrm{W}_{1}, \mathrm{~W}_{2}\right)$ we input $\left(\mathrm{L}, \mathrm{W}_{1}, \mathrm{~W}_{1}\right)$ into the $\mathrm{LP}$.

The watermark extraction process is summarized in Figure 7.

By increasing the levels of decomposition, the watermarking capacity is also increased, and the quality of

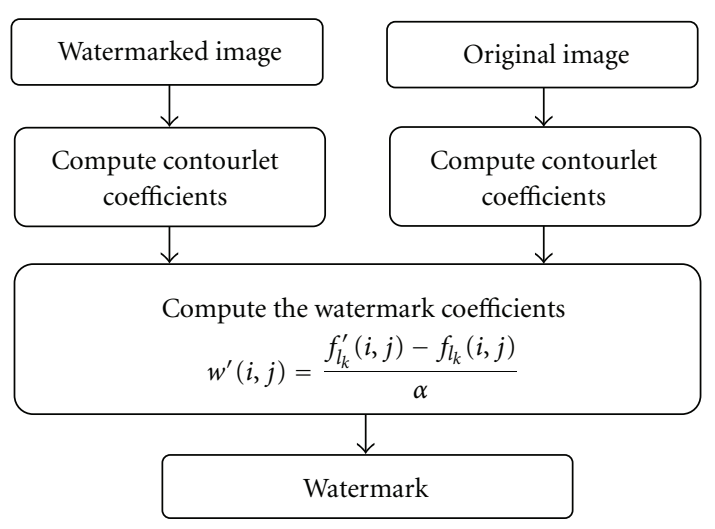

Figure 7: Extraction algorithm.

extracted watermark is improved. In order to achieve this goal, after selecting a subband, we can use other directional subbands which have the highest level of energy. The watermarked image quality is measured by the PSNR between $f$ and $f^{\prime}$, formulated by

$$
\begin{gathered}
\text { PSNR }=10 \log _{10}\left(\frac{255^{2}}{\mathrm{MSE}}\right)(\mathrm{dB}), \\
\mathrm{MSE}=\frac{1}{M \times N} \sum_{i=1}^{M} \sum_{j=1}^{N}\left(f(i, j)-f^{\prime}(i, j)\right)^{2} .
\end{gathered}
$$

To evaluate the performance of watermark retrieval process, normalized correlation (NC) is used. Here, $W_{1}$ and $W_{2}$ are the original and recovered watermark signals, respectively. The normalized correlation is calculated by

$$
\mathrm{NC}=\left(\frac{\sum_{i=0}^{M} \sum_{j=0}^{N} W_{1}(i, j) \cdot W_{2}(i, j)}{\left(\sqrt{\sum_{i=0}^{M} \sum_{j=0}^{N} W_{1}(i, j)^{2}}\right) \cdot\left(\sqrt{\sum_{i=0}^{M} \sum_{j=0}^{N} W_{2}(i, j)^{2}}\right)}\right) .
$$

\section{Experimental Results}

We have performed experiments with various watermarks and popular host images such as Lena, Barbara, Baboon, Cameraman, City, Couple, Man, Boat, Elaine, Peppers, and Zelda of size $512 \times 512$. The watermark is a grayscale fingerprint (.bmp) of size $128 \times 128$, which contains lots of curves and significant details. Therefore, it can be a perfect criterion for measuring the performance of the proposed method. In addition, it can be used in fingerprinting applications. In (2), $\alpha$ was set to 0.1 to obtain a tradeoff between perceptibility and robustness. In both LP and DFB decomposition, "PKVA" filters [18] were used because of their efficient implementation. We decomposed the host image into four levels, and the watermark into one level.

4.1. Watermark Invisibility. Figures $8(\mathrm{a})$ and $8(\mathrm{~b})$ provide the comparison between the original Lena test image and its corresponding watermarked image.The original watermark and the extracted watermark are also shown in Figures 8(c) 


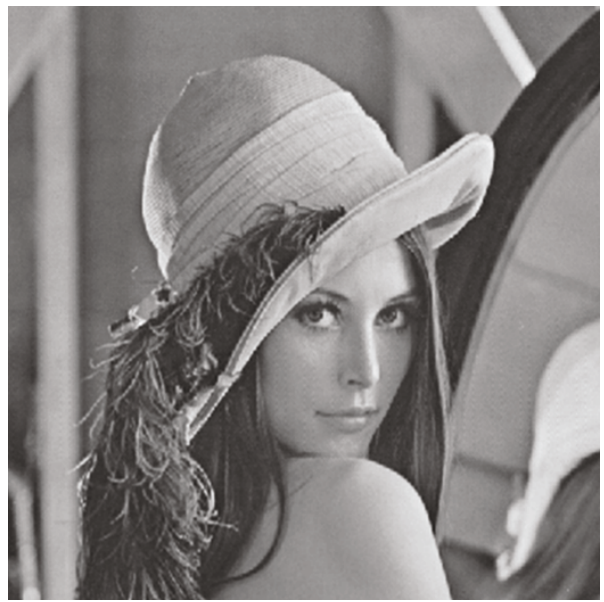

(a)

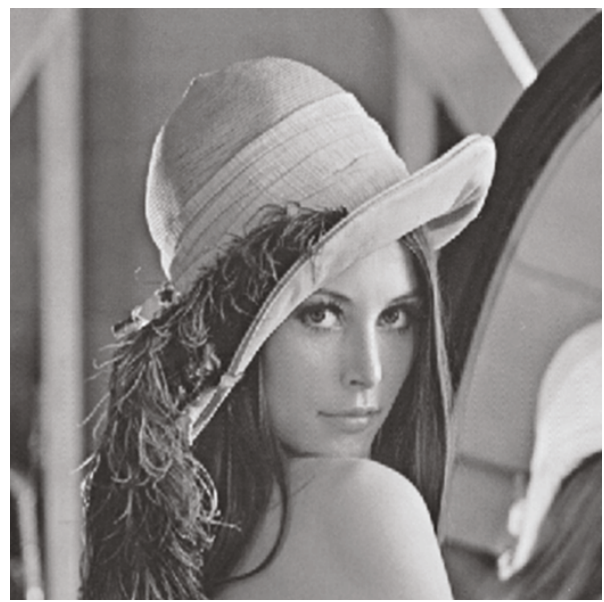

(b)

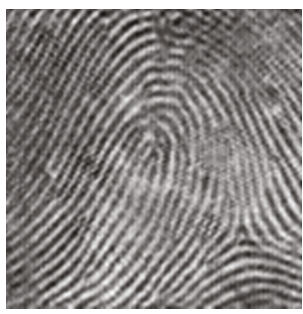

(c)

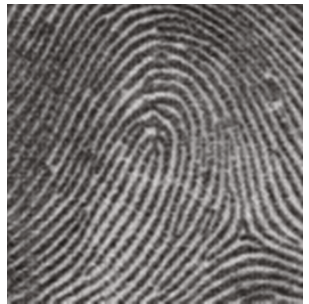

(d)

FIgURE 8: (a) Lena image. (b) Watermarked image. (c) Original watermark. (d) Extracted watermark.

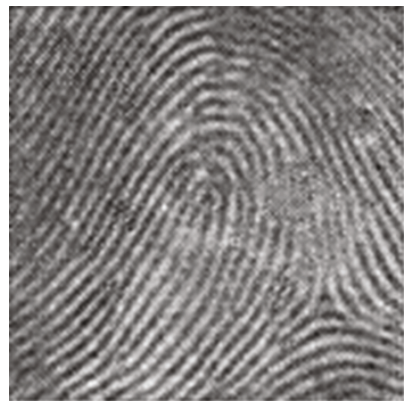

(a)

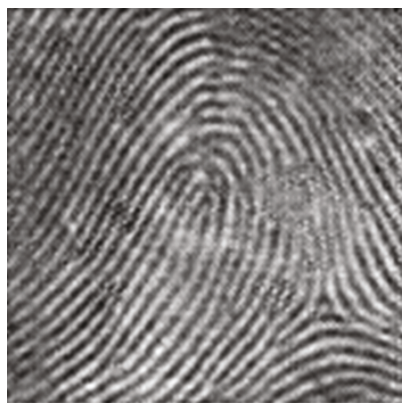

(b)

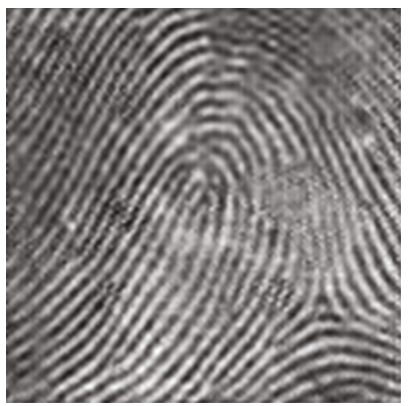

(c)

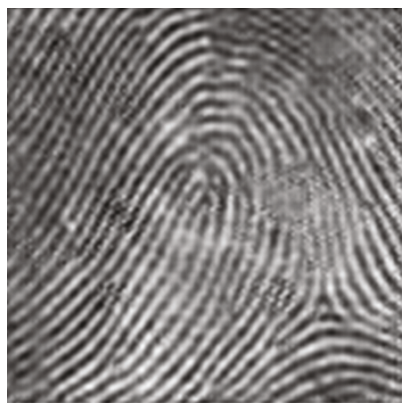

(d)

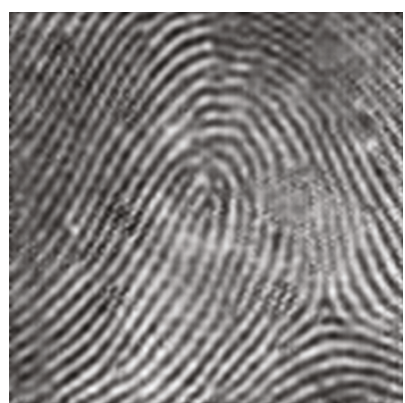

(e)

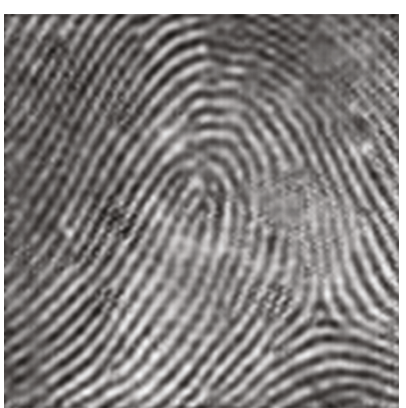

(f)

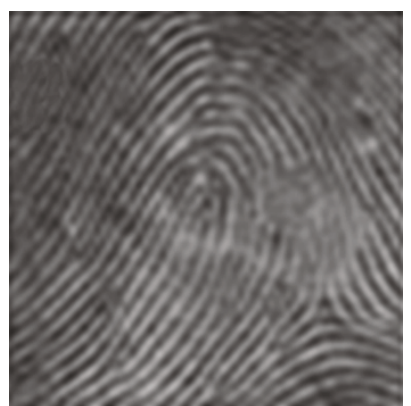

(g)

FiguRE 9: Recovered watermarks from Lena image after JPEG2000 compression. (a) Rate $=0.3$ (b) Rate $=0.4$ (c) Rate $=0.5$ (d) Rate $=0.6$ (e) Rate $=0.7$ (f) Rate $=0.8(\mathrm{~g})$ Rate $=0.9$. 


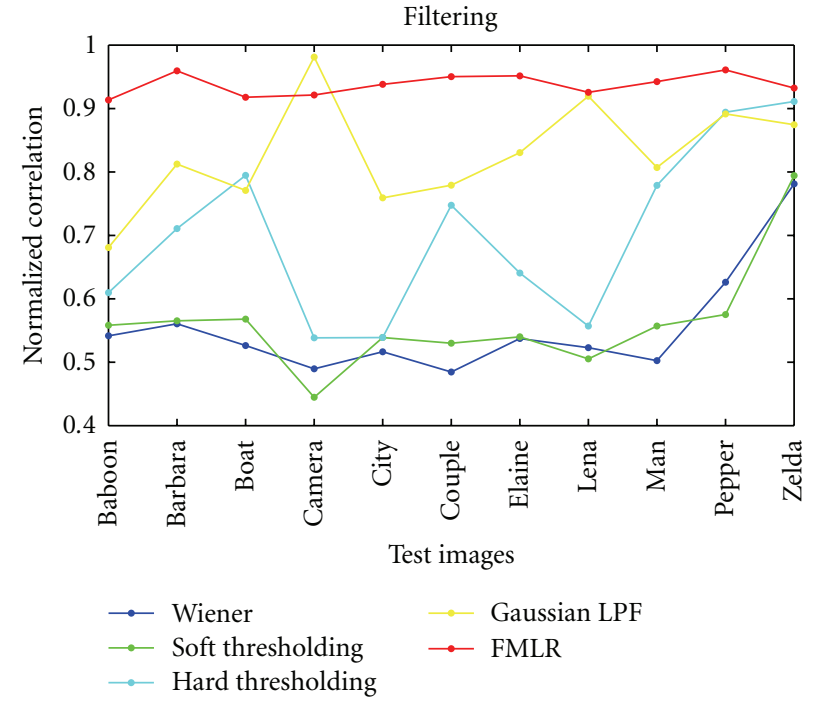

(a)

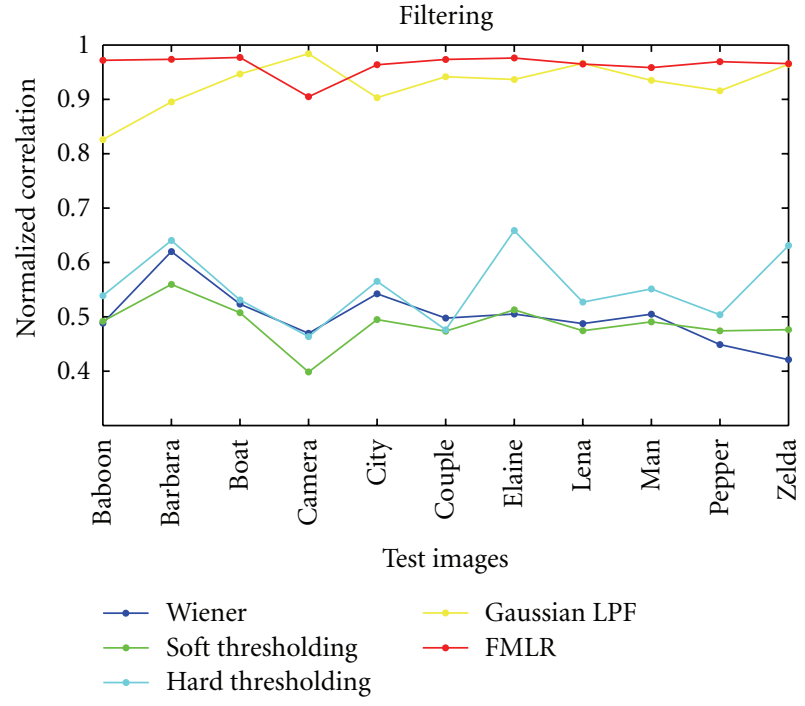

(b)

FIGURE 10: Normalized correlation results of different test images under different filtering attacks (window size $=3 \times 3$ ) (a) embedding the watermark in the highest frequency subband of the host image (b) embedding the watermark in the 16th directional subband.

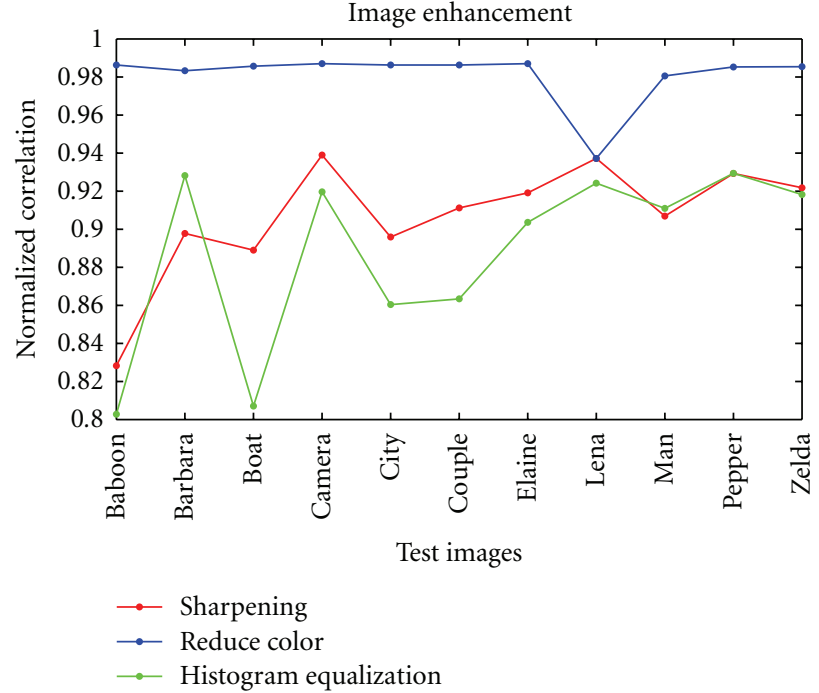

(a)

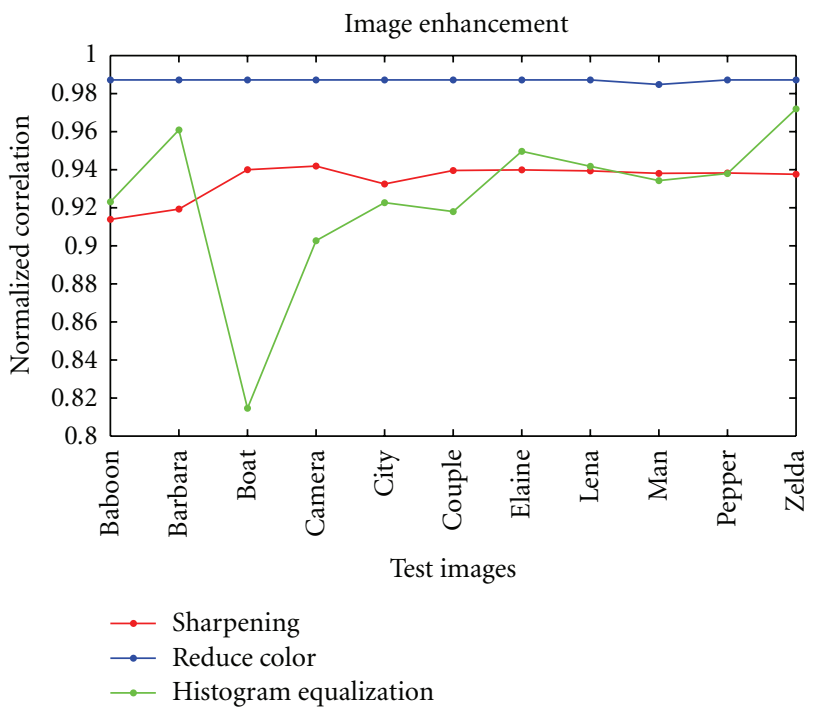

(b)

FIGURE 11: Normalized correlation results of different test images under image enhancement attacks (a) embedding the watermark in the highest frequency subband of the host image (b) embedding the watermark in the 16th directional subband.

and $8(\mathrm{~d})$, respectively. The results of embedding data in the highest frequency subband of the host image are shown in Table 1.

Our experiments on the test images showed that the 16th directional subbands have the highest priority for watermark embedding. The results of embedding the watermark in the 16th directional subbands of the host images were as follows. The watermark invisibility can be guaranteed at average PSNR value of $46.96 \mathrm{~dB}$ for all the test images due to their similar characteristics and the NC value of 0.9862 for all the extracted watermarks except for the Man image, for which the PSNR and NC values were 47.09 and 0.9838 , respectively.
The results of hiding more amounts of data into the highest and other directional subbands of the Lena test image are shown in Table 2. The PSNR and NC values for other subbands are also shown in columns 2 and 3 of the same table, respectively. We used the 1st and the 4th directional subbands that have the highest level of energy after the 16th subband. In addition to embedding the watermark into the 16th directional subband, we hide another version of the watermark into the 1st and the 4th subband, and thus we could embed $34 \mathrm{~KB}$ of data into the host image without degrading its perceptual quality. Embedding the watermark in other subbands with lower energy than a given threshold 


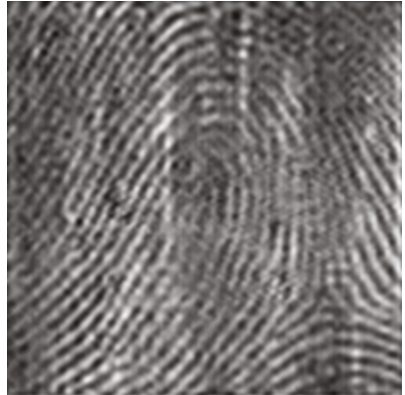

(a)

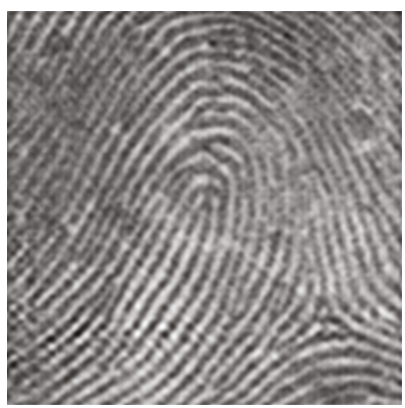

(e)

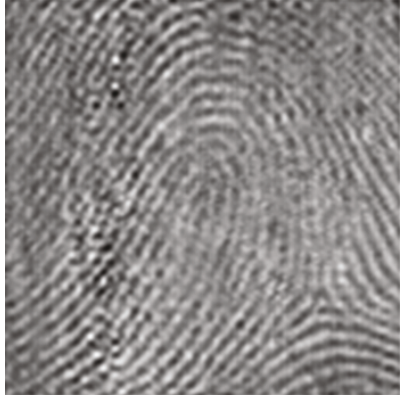

(b)

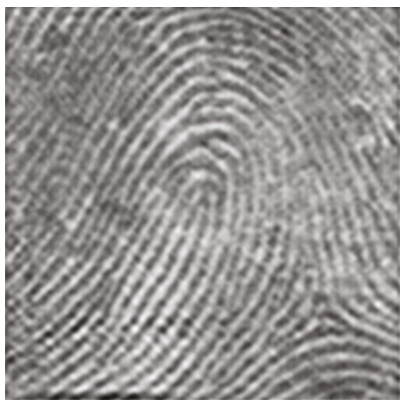

(f)

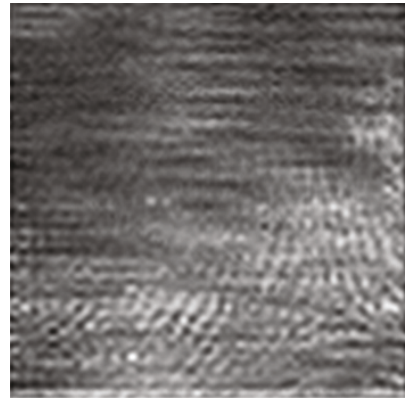

(c)

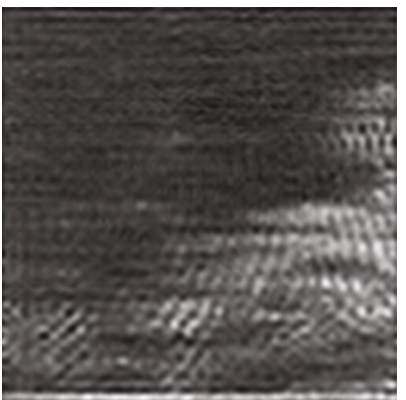

(g)

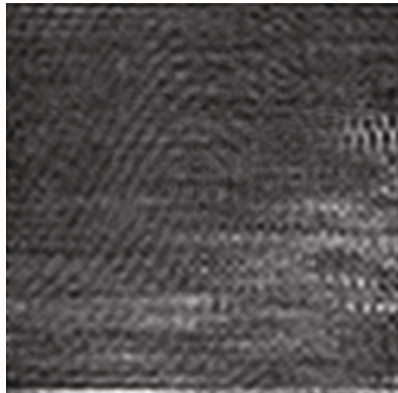

(d)

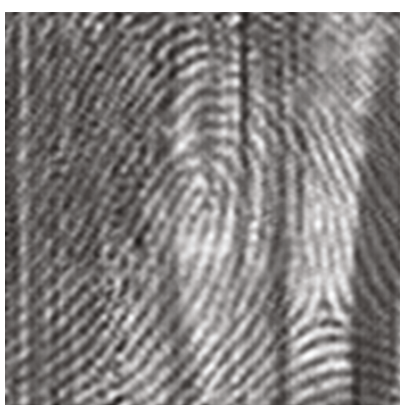

(h)

FIGURE 12: Recovered watermarks from Lena image under various filtering and enhancement attacks (a) FMLR, (b) Gaussian LPF, (c) hard thresholding (d) soft thresholding (e) reduce color (f) image sharpening (g) Wiener filtering (h) histogram equalization.

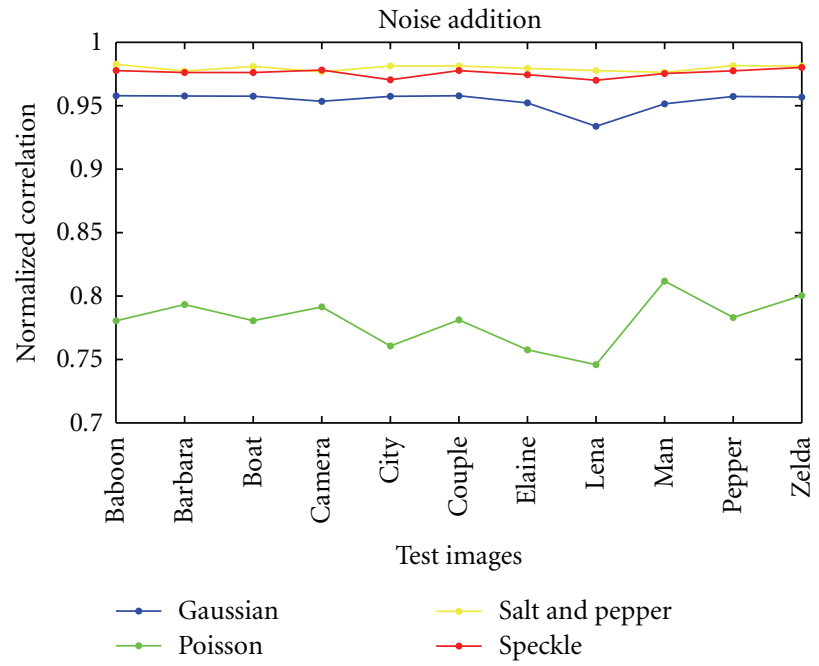

(a)

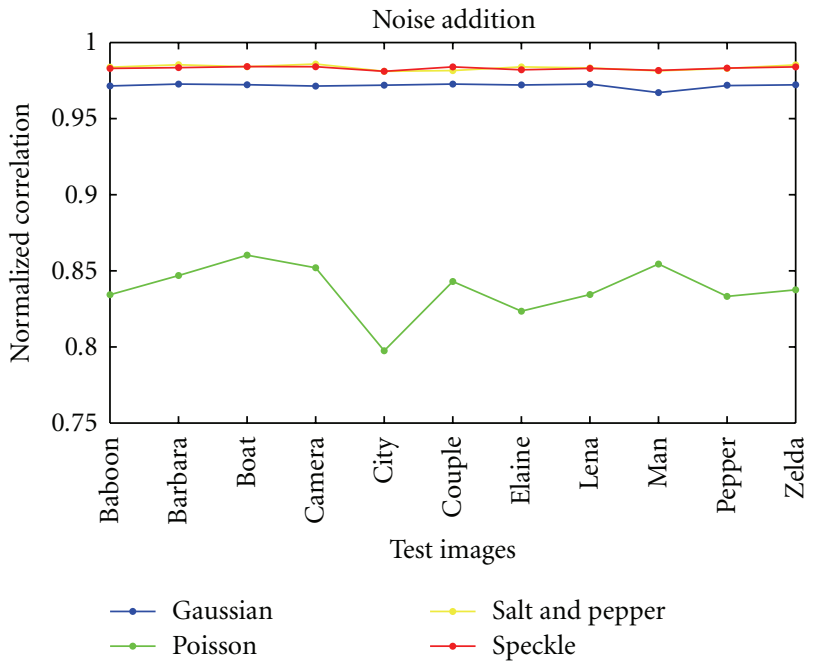

(b)

FIGURE 13: Normalized correlation results of different test images under noise attacks (a) embedding the watermark in the highest frequency subband of the host image (b) embedding the watermark in the 16th directional subband.

will result in perceptual distortion in the watermarked image. Table 3 shows the results of embedding data in the Lena test image with different sizes. The size of the watermark is $25 \%$ of the size of the host image.

4.2. Resistance to Various Attacks. It is known that embedding the watermark at the high-frequency subbands of an image is sensitive to many image processing algorithms such as lowpass filtering, lossy compression, noise, and geometrical distortion. On the other hand, the watermark at low-frequency subbands of an image is sensitive to other image processing algorithms such as histogram equalization and cropping. As we mentioned in Section 3, although the watermark is embedded into the highest frequency subbands, it is likely to be spread out into all subbands when we reconstruct the watermarked image, due to the special 


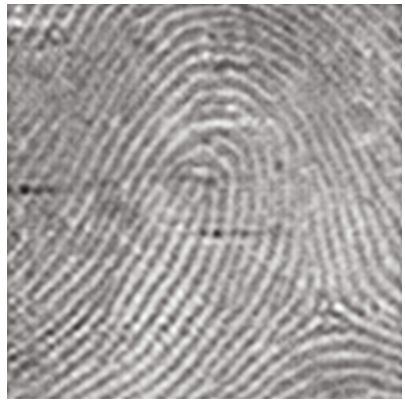

(a)

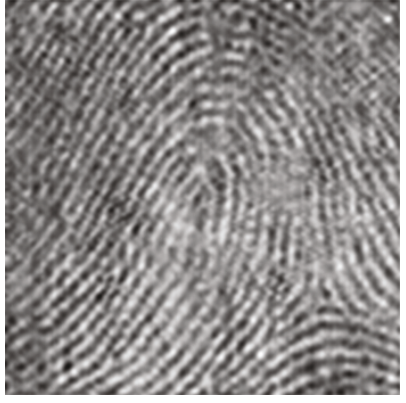

(b)

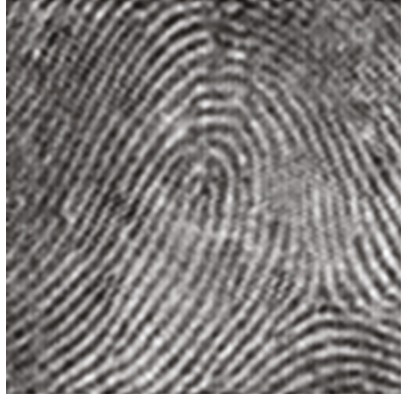

(c)

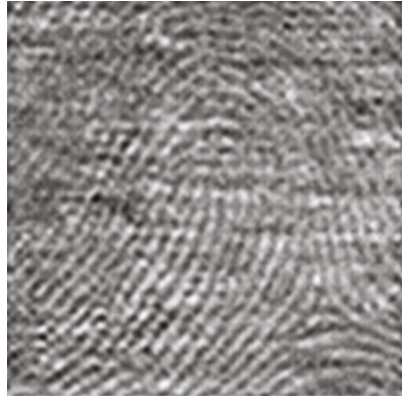

(d)

FIGURE 14: Recovered watermarks from Lena image after applying different noises (a) Salt and pepper (density $=0.0001)(\mathrm{b})$ Gaussian noise $($ density $=0.0001)(\mathrm{c})$ Speckle noise $($ density $=0.0001)(\mathrm{d})$ Poisson noise.

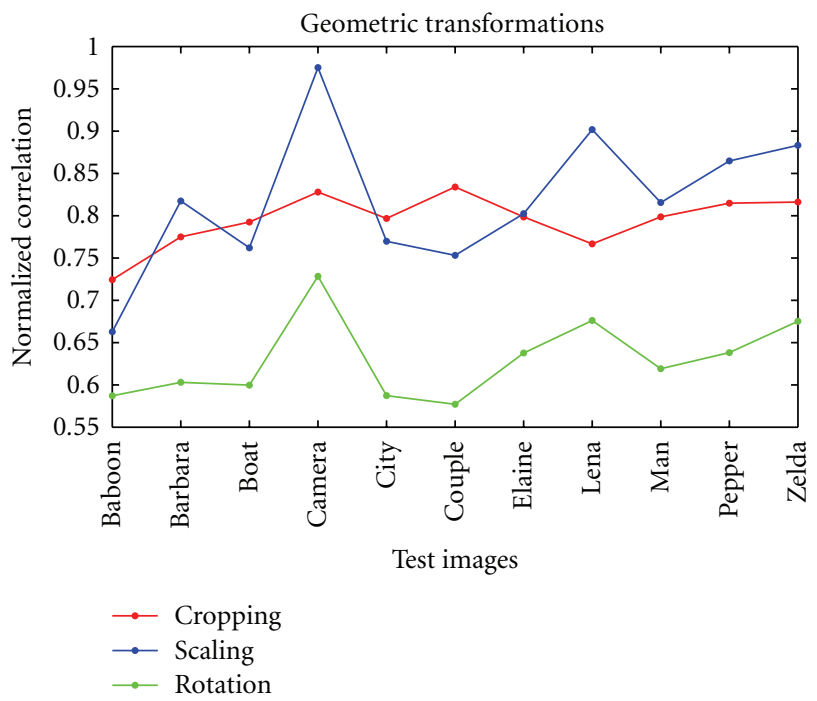

(a)

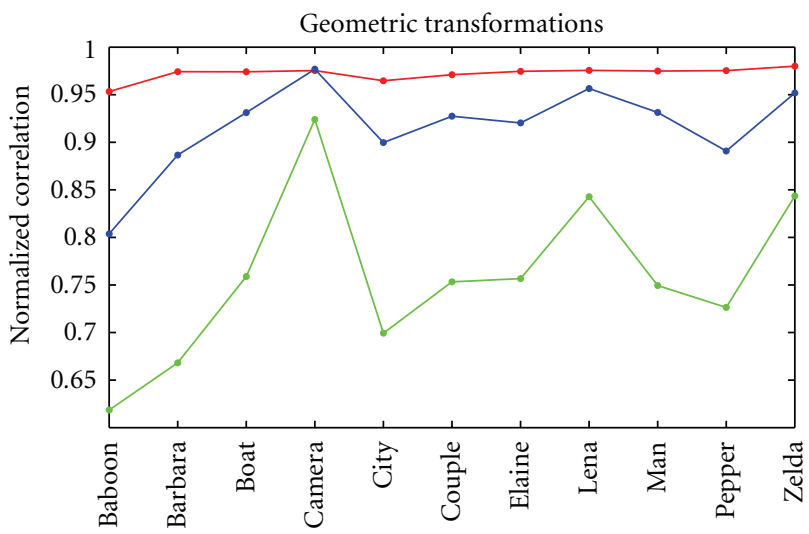

Test images

$$
\begin{aligned}
& \because \text { Cropping } \\
& \because \text { Scaling } \\
& \because \text { Rotation }
\end{aligned}
$$

(b)

FIGURE 15: Normalized correlation results of different test images under geometrical attacks: (a) embedding in the highest frequency subband of the host image (b) embedding the watermark in the 16th directional subband.

TABLE 1: Results of embedding data in the highest frequency subband of the host image.

\begin{tabular}{lccc}
\hline Host image & Highest frequency subband & PSNR & NC \\
\hline Baboon & 13 & 37.0757 & 0.986389 \\
Barbara & 6 & 36.7178 & 0.983328 \\
Boat & 4 & 36.7234 & 0.985742 \\
Cameraman & 1 & 45.7128 & 0.987057 \\
City & 13 & 37.0794 & 0.98637 \\
Couple & 13 & 37.0754 & 0.986388 \\
Elaine & 1 & 45.7083 & 0.987072 \\
Lena & 16 & 46.968 & 0.986253 \\
Man & 4 & 36.9369 & 0.98061 \\
Peppers & 4 & 36.7585 & 0.985308 \\
Zelda & 4 & 36.7616 & 0.985401 \\
\hline
\end{tabular}

transform structure of the Laplacian Pyramid. In this section, we attempt to show the robustness of our watermarking
TABLE 2: Results of embedding more amounts of data into 16th and another directional subband.

\begin{tabular}{lcc}
\hline Subband & PSNR & $\mathrm{NC}$ \\
\hline $16 \& 1$ & 43.3194 & $\mathrm{NC}_{16}=0.9596$ \\
& $\mathrm{NC}_{1}=0.9852$ \\
\hline \multirow{2}{*}{$16 \& 4$} & 36.3084 & $\mathrm{NC}_{16}=0.8954$ \\
& & $\mathrm{NC}_{4}=0.9858$ \\
\hline
\end{tabular}

TABle 3: Results of embedding data in Lena image with different size.

\begin{tabular}{lccc}
\hline Host image size & Watermark size & PSNR $(\mathrm{dB})$ & NC \\
\hline $1024 \times 1024$ & $256 \times 256(65 \mathrm{~KB})$ & 47.1197 & 0.996708 \\
$512 \times 512$ & $128 \times 128(17 \mathrm{~KB})$ & 46.968 & 0.986253 \\
$256 \times 256$ & $64 \times 64(5.05 \mathrm{~KB})$ & 36.9065 & 0.977844 \\
\hline
\end{tabular}

scheme for both high-and low-frequency, signal processing attacks. The MATLAB 7.0 and Checkmark 1.2 [19] were 
TABLE 4: Normalized correlation coefficients after JPEG2000 compression on watermarked images in which the watermark is embedded in the highest frequency subband.

\begin{tabular}{|c|c|c|c|c|c|c|c|}
\hline Host & 0.3 & 0.4 & 0.5 & 0.6 & 0.7 & 0.8 & 0.9 \\
\hline Baboon & 0.907042 & 0.954985 & 0.973306 & 0.978915 & 0.983985 & 0.98639 & 0.98639 \\
\hline Barbara & 0.971182 & 0.977175 & 0.980079 & 0.98293 & 0.983295 & 0.983295 & 0.983295 \\
\hline Boat & 0.96877 & 0.976222 & 0.980652 & 0.985366 & 0.985643 & 0.985643 & 0.985643 \\
\hline Cameraman & 0.983941 & 0.986697 & 0.987041 & 0.987041 & 0.987041 & 0.987041 & 0.987041 \\
\hline City & 0.955788 & 0.973253 & 0.981524 & 0.984497 & 0.986288 & 0.986288 & 0.986288 \\
\hline Couple & 0.971682 & 0.980032 & 0.983572 & 0.986312 & 0.986312 & 0.986312 & 0.986312 \\
\hline Elaine & 0.964434 & 0.980293 & 0.984266 & 0.98591 & 0.986947 & 0.986947 & 0.986947 \\
\hline Lena & 0.97721 & 0.982396 & 0.985192 & 0.986252 & 0.986252 & 0.986252 & 0.986252 \\
\hline Man & 0.9623 & 0.971662 & 0.975141 & 0.980085 & 0.980611 & 0.980611 & 0.980611 \\
\hline Peppers & 0.967675 & 0.977996 & 0.982511 & 0.985192 & 0.985192 & 0.985192 & 0.985192 \\
\hline Zelda & 0.977523 & 0.980563 & 0.985064 & 0.985319 & 0.985319 & 0.985319 & 0.985319 \\
\hline
\end{tabular}

TABLE 5: Normalized correlation coefficients after JPEG2000 compression on watermarked images in which the watermark is embedded in the 16th directional subband.

\begin{tabular}{lccccccc}
\hline Host & 0.3 & 0.4 & 0.5 & 0.6 & 0.7 & 0.8 & 0.9 \\
\hline Baboon & 0.9407 & 0.9770 & 0.9836 & 0.9854 & 0.9865 & 0.9871 & 0.9871 \\
Barbara & 0.9824 & 0.9846 & 0.9860 & 0.9869 & 0.9871 & 0.9871 & 0.9871 \\
Boat & 0.9766 & 0.9841 & 0.9863 & 0.9870 & 0.9872 & 0.9872 & 0.9872 \\
Cameraman & 0.9850 & 0.9864 & 0.9872 & 0.9872 & 0.9872 & 0.9872 & 0.9872 \\
City & 0.9730 & 0.9837 & 0.9852 & 0.9865 & 0.9872 & 0.9872 & 0.9872 \\
Couple & 0.9835 & 0.9849 & 0.9864 & 0.9872 & 0.9872 & 0.9872 & 0.9872 \\
Elaine & 0.9792 & 0.9850 & 0.9861 & 0.9869 & 0.9872 & 0.9872 & 0.9872 \\
Lena & 0.9842 & 0.9862 & 0.9869 & 0.9872 & 0.9872 & 0.9872 & 0.9872 \\
Man & 0.9697 & 0.9798 & 0.9832 & 0.9845 & 0.9848 & 0.9848 & 0.9848 \\
Peppers & 0.9832 & 0.9851 & 0.9864 & 0.9872 & 0.9872 & 0.9872 & 0.9872 \\
Zelda & 0.9840 & 0.9863 & 0.9869 & 0.9871 & 0.9871 & 0.9871 & 0.9871 \\
\hline
\end{tabular}

TABLE 6: Comparison of the proposed method with other domain methods.

\begin{tabular}{llll}
\hline Characteristic & $\begin{array}{l}\text { Proposed } \\
\text { method }\end{array}$ & $\begin{array}{l}\text { Elbasi \& } \\
\text { Eskicioglu's } \\
\text { Method }\end{array}$ & $\begin{array}{l}\text { Wang \& } \\
\text { Pearmain } \\
\text { Method }\end{array}$ \\
\hline $\begin{array}{l}\text { Transform } \\
\text { domain }\end{array}$ & Contourlet & Wavelet & DCT \\
\hline Watermark type & Gray scale & PRN sequence & Binary \\
\hline $\begin{array}{l}\text { No. watermark } \\
\text { bits embedded }\end{array}$ & $17 \mathrm{~KB}(128 \times 128)$ & - & 910 \\
\hline $\begin{array}{l}\text { PSNR in dB } \\
\text { No. reported }\end{array}$ & 46.97 & 40.86 & 39.21 \\
\hline attacks & 13 & 9 & 4 \\
\hline Extraction type & Nonblind & Semiblind & Blind \\
\hline
\end{tabular}

used for testing the robustness of the proposed method. The wide class of existing attacks can be divided into four main categories: removal attacks, geometrical attacks, cryptographic attacks, and protocol attacks [20]. We investigate the robustness of our method against removal and geometrical attacks.

4.2.1. Removal Attacks. Removal attacks aim at the complete removal of the watermark information from the watermark data without cracking the security of the watermarking algorithm [20]. To test the robustness of our method against removal attacks JPEG2000 compression, image enhancement techniques, various noise, and filtering attacks were used.

The JPEG2000 attack was tested using Jasper 1.900.1 [21]. Table 4 shows the results of applying JPEG2000 attack on the watermarked images in which the watermark is embedded in the highest frequency subband of the host image and Table 5 shows the results of applying JPEG2000 attack on watermarked images in which the watermark is embedded in the 16th directional subband of the host image. The results demonstrate an excellent robustness of our method against JPEG2000 compression. Figure 9 shows the extracted 


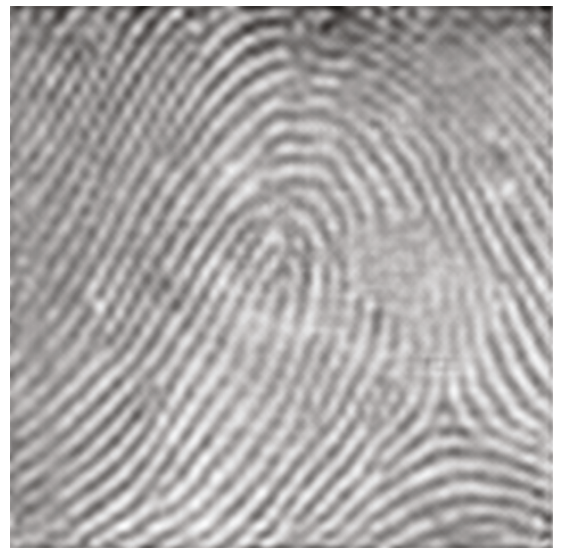

(a)

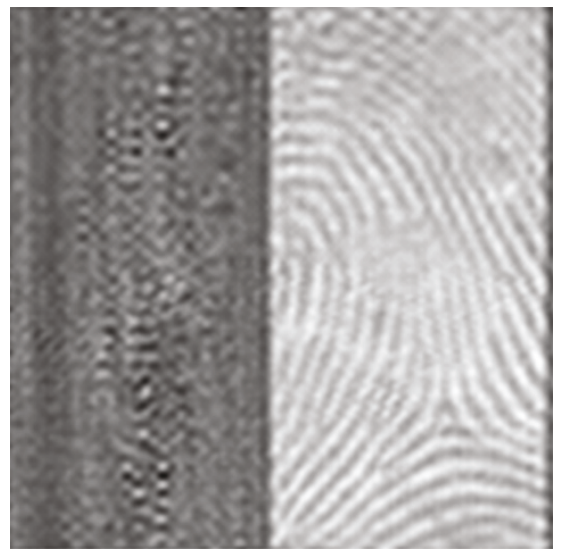

(d)

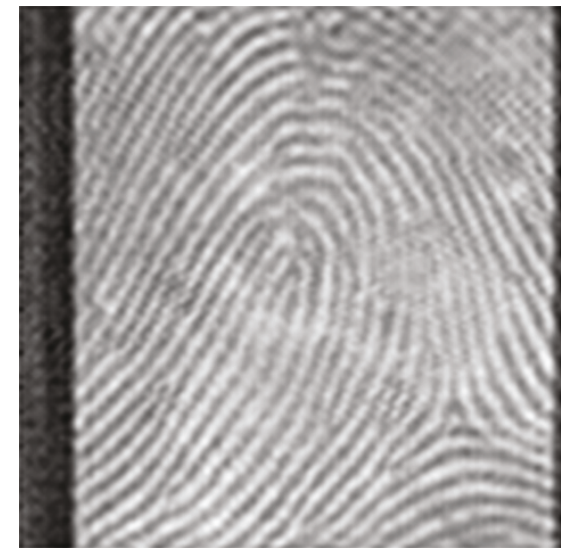

(b)

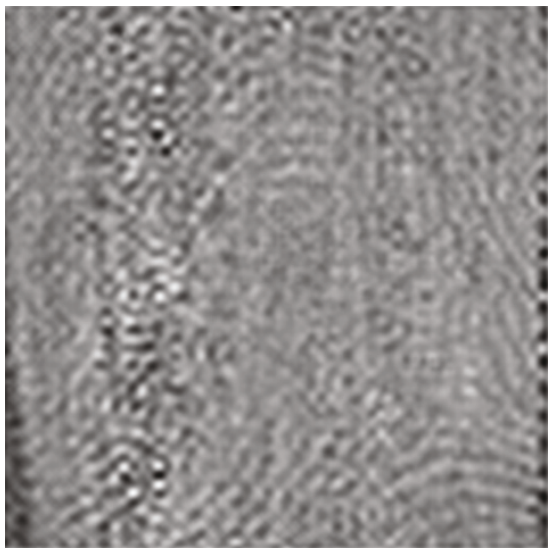

(e)

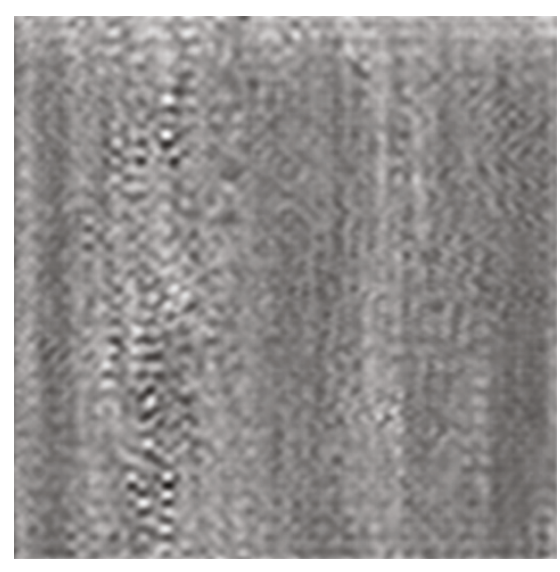

(g)

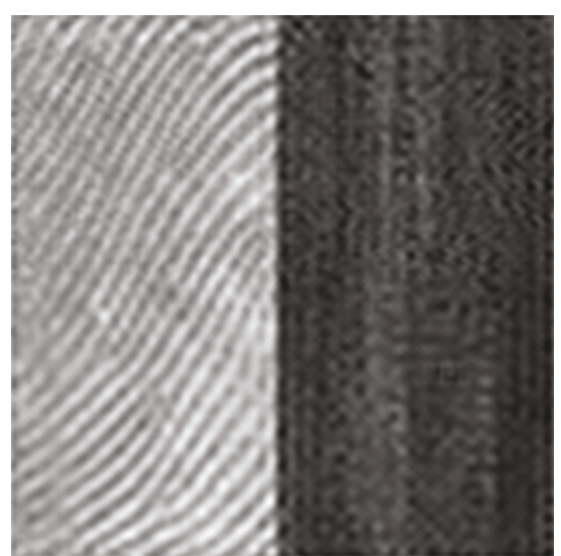

(c)

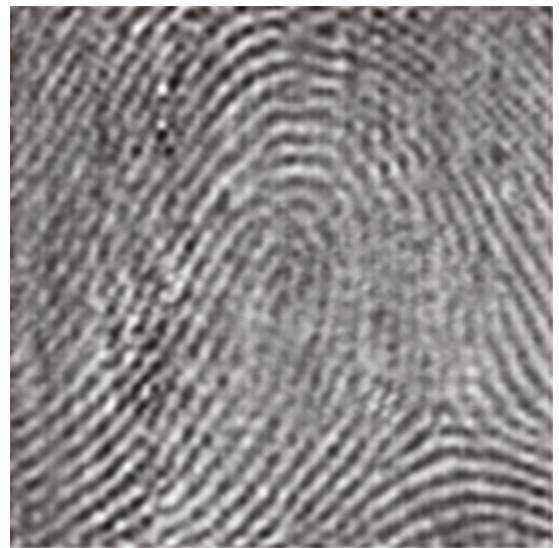

(f) 


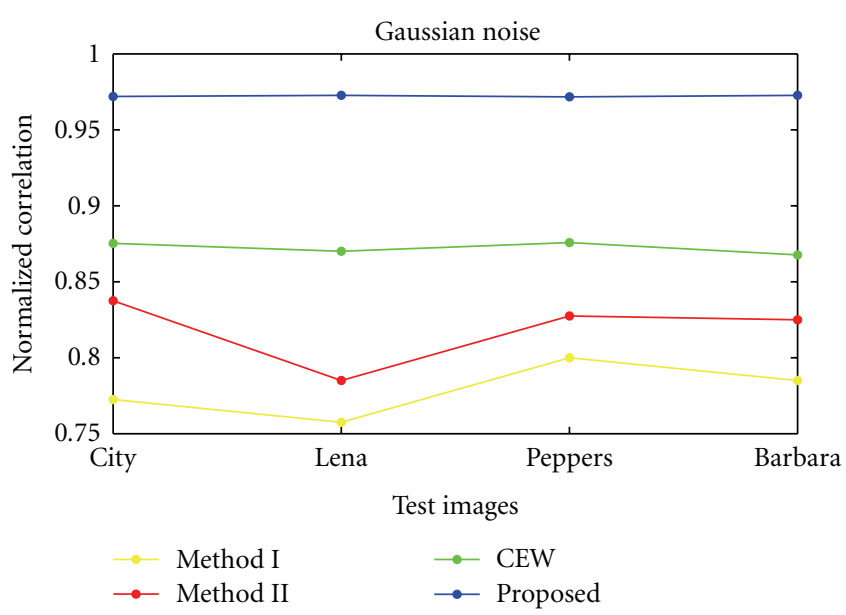

Figure 17: The robustness comparison result of the proposed method with $[2,3]$ under Gaussian attack (mean, var) $=(0$, 0.0001).

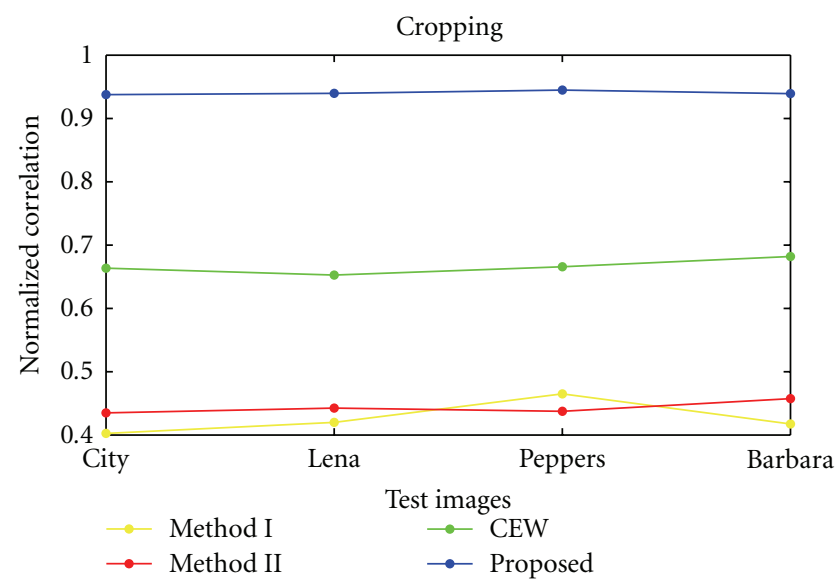

FIGURE 18: The robustness comparison result of the proposed method with $[2,3]$ under cropping attack $(400 \times 450)$.

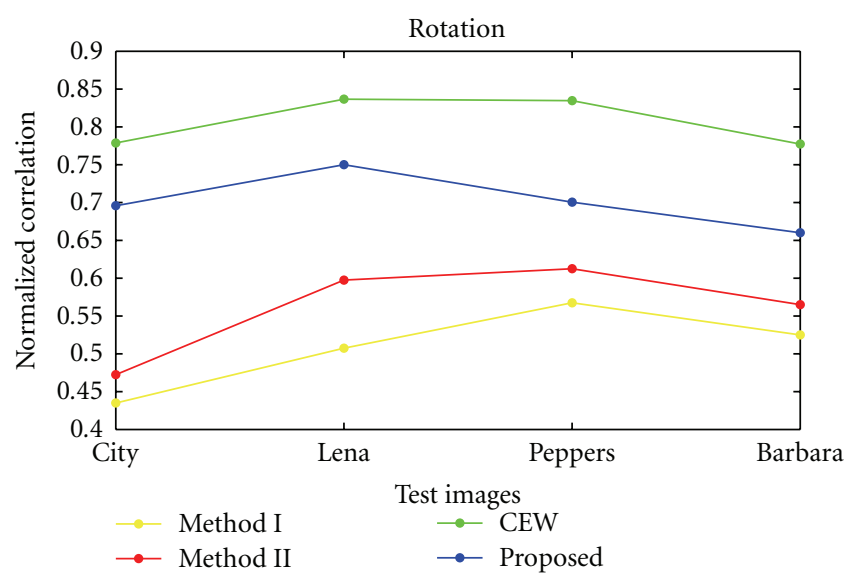

Figure 19: The robustness comparison result of the proposed method with $[2,3]$ under rotation attack $\left(\right.$ angle $\left.=6^{\circ}\right)$.
TABLE 7: Comparison of the proposed method with similar domain methods.

\begin{tabular}{llll}
\hline Characteristic & $\begin{array}{l}\text { Proposed } \\
\text { method }\end{array}$ & CEW & $\begin{array}{l}\text { Method I \& } \\
\text { Method II }\end{array}$ \\
\hline Watermark type & Gray scale & Binary & Binary \\
\hline $\begin{array}{l}\text { No. watermark } \\
\text { bits embedded }\end{array}$ & $17 \mathrm{~KB}(128 \times 128)$ & Not mentioned & $16 \times 16$ \\
\hline PSNR in dB & 46.97 & Not mentioned & $\approx 47$ \\
\hline $\begin{array}{l}\text { No. reported } \\
\text { attacks }\end{array}$ & 13 & 3 & 3 \\
\hline Extraction type & Nonblind & Nonblind & Nonblind \\
\hline
\end{tabular}

Figure 12 shows the recovered watermarks of the Lena test image under these attacks. The results show good robustness properties of the proposed method against all the tested attacks except for the thresholding and Wiener filtering.

To test the robustness of our method under various noise processes, Gaussian noise, salt \& pepper noise, speckle noise, and Poisson noise with a density of 0.0001 were used. Figure 13 shows the normalized correlation coefficient results of applying various noise attacks on different test images. Figure 14 shows the recovered watermarks of the Lena test image under different noise processes. Results demonstrate excellent resistance of our method against common noises.

4.2.2. Geometrical Attacks. In contrast to removal attacks, geometrical attacks do not actually remove the embedded watermark itself but intend to distort the watermark detector synchronization with the embedded information [20]. The most common geometrical attacks are rotation, scaling, and cropping. The parameters used in these attacks are a rotation angle of $20^{\circ}$, a scaling factor of 2, and cropping size of $256 \times 512$ (the top half is removed). Figure 15 illustrates the normalized correlation coefficient results of these attacks on different test images. Figure 16 shows the extracted watermarks after applying geometric attacks on the Lena test image. Results of cropping other parts of the Lena test image are also shown in Figure 16. Results demonstrate good resistance of our method against cropping and scaling but poor resistance against rotation attack.

4.3. Comparison. The performance of the proposed method was compared with 2 other methods with two different decomposition types and the results are shown in Table 6 . Wang and Pearmain's method [22] is a blind watermarking technique based on the patch work estimation. A total of 910 watermark bits were embedded in the Lena test image by using DCT. The PSNR reported was $39.21 \mathrm{~dB}$ and the numbers of attacks reported were only 4 . Elbasi and Eskicioglu's method [23] is a semiblind DWT watermarking technique which embeds a pseudorandom number (PRN) sequence as a watermark in three bands of the image, using coefficients that are higher than a given threshold. The reported PSNR was $40.86 \mathrm{~dB}$ and the numbers of 
attacks reported were 9. In the proposed method, $17 \mathrm{~KB}$ are embedded and the obtained PSNR is $46.97 \mathrm{~dB}$. The Watermarked image in our method can survive many attacks, and it is superior in terms of PSNR compared to these methods. Furthermore, we compared our method with three related works, which also used contourlet decomposition. Method I and Method II are reported in [3], and CEW is reported in [2]. Table 7 summarizes the comparison results of the proposed method with these methods. Figures 17, 18 and 19 show the comparison results between Method I, Method II, CEW, and the proposed method on the popular test images under the Gaussian noise, cropping, and rotation attack, respectively. In the Gaussian noise and cropping attacks, our method outperforms other methods but in rotation attack (angle $=6^{\circ}$ ), the performance of CEW was better.

\section{Conclusion}

In this paper, we proposed a new multiresolution watermarking method using the contourlet transform. In this method, a grayscale watermark was added to the highest frequency subband of the host image. The quality of the watermarked image was good in terms of perceptibility and PSNR (average of $39.4107 \mathrm{~dB}$ ) measures. We showed that we can embed a remarkable amount of data $(34 \mathrm{~KB})$ using other high frequency subbands in addition to the highest frequency subband. Moreover, we showed that this method was robust against various removal and geometrical attacks such as JPEG2000 compression, salt and pepper noise, Gaussian noise, speckle noise, Poisson noise, frequency mode Laplacian removal, Gaussian lowpass filtering, reduced color, image sharpening, cropping, scaling, and histogram equalization. We compared the robustness of the proposed method with 3 other contourlet methods under cropping, Gaussian noise, and rotation attacks. Compared to the DWT-based and DCT-based methods, the proposed method is superior in terms of embedding capacity, PSNR and survival to a number of image attacks. Considering the good characteristics of our method such as imperceptibility, robustness and non-blind extraction, it would be a suitable choice for fingerprinting applications. Our future focus will be on enhancing the robustness properties of the proposed algorithm against various attacks.

\section{References}

[1] J. Cox, M. L. Miller, and J. A. Bloom, "Watermarking applications and their properties," in Proceedings of the International Conference on Information Technology: Coding and Computing (ITCC '00), pp. 6-10, Las Vegas, Nev, USA, 2000.

[2] S. Zaboli and M. S. Moin, "CEW: A non-blind adaptive image watermarking approach based on entropy in contourlet domain," in 2007 IEEE International Symposium on Industrial Electronics, ISIE 2007, pp. 1687-1692, esp, June 2007.

[3] M. Jayalakshmi, S. N. Merchant, and U. B. Desai, "Digital watermarking in contourlet domain," in 18th International Conference on Pattern Recognition, ICPR 2006, pp. 861-864, chn, August 2006.
[4] G. Duan, A.T. S. Ho, and X. Zhao, "A Novel non-redundant contourlet transform for robust image watermarking against non-geometrical and geometrical attacks," in Proceedings of the 5th International Conference on Visual Information Engineering (VIE '08), pp. 124-129, August 2008.

[5] S. Xiao, H. Ling, F. Zou, and Z. Lu, "Adaptive image watermarking algorithm in contourlet domain," in 2007 JapanChina Joint Workshop on Frontier of Computer Science and Technology, FCST 2007, pp. 125-130, chn, November 2007.

[6] E. Salahi, M. S. Moin, and A. Salahi, "A new visually imperceptible and robust image watermarking scheme in Contourlet domain," in 2008 4th International Conference on Intelligent Information Hiding and Multiedia Signal Processing, IIH-MSP 2008, pp. 457-460, chn, August 2008.

[7] Z. Shu, S. Wang, C. Deng, G. Liu, and L. Zhang, "Watermarking algorithm based on contourlet transform and human visual model," in 2008 International Conference on Embedded Software and Systems, ICESS-08, pp. 348-352, chn, July 2008.

[8] X. Lian, X. Ding, and D. Guo, "Digital watermarking based on non-sampled contourlet transform," in 2007 IEEE International Workshop on Anti-counterfeiting, Security, Identification, ASID, pp. 138-141, chn, April 2007.

[9] F. Wei, T. Ming, and J. Hong-Bing, "An adaptive watermark scheme based on contourlet transform," in International Symposium on Computer Science and Computational Technology, ISCSCT 2008, pp. 677-681, chn, December 2008.

[10] P. Meerwald and A. Uhl, "A survey of wavelet domain watermarking algorithms," in Electronic Imaging, Security and Watermarking of Multimedia Contents, vol. 4314 of Proceedings of SPIE, January 2001.

[11] D. Kundur and D. Hatzinakos, "Towards robust logo watermarking using multiresolution image fusion principles," IEEE Transactions on Image Processing, vol. 6, no. 1, pp. 185-198, 2004.

[12] M. N. Do and M. Vetterli, "The contourlet transform: An efficient directional multiresolution image representation," IEEE Transactions on Image Processing, vol. 14, no. 12, pp. 2091-2106, 2005.

[13] S. Khalighi, P. Tirdad, and H. R. Rabiee, "A new robust nonblind digital watermarking scheme in contourlet domain," in Proceedings of the 9th IEEE International Symposium on Signal Processing and Information Technology (ISSPIT '09), Ajman ,UAE, December 2009.

[14] D. D.-Y. Po and M. N. Do, "Directional multiscale modeling of images using the contourlet transform," IEEE Transactions on Image Processing, vol. 15, no. 6, pp. 1610-1620, 2006.

[15] M. N. Do and M. Vetterli, "Pyramidal directional filter banks and curvelets," in Proceedings of IEEE International Conference on Image Processing (ICIP '01), vol. 3, pp. 158-161, Thessaloniki, Greece, October 2001.

[16] M. N. Do and M. Vetterli, "Framing pyramids," IEEE Transactions on Signal Processing, vol. 51, no. 9, pp. 2329-2342, 2003.

[17] I. J. Cox, J. Kilian, T. Leighton, and T. G. Shamoon, "Secure spread spectrum watermarking for multimedia," in Proceedings of IEEE International Conference on Image Processing (ICIP'97), vol. 6, pp. 1673-1687, Santa Barbara, Calif, USA, October 1997.

[18] S. Phoong, C. W. Kim, P. P. Vaidyanathan, and R. Ansari, "New class of two-channel biorthogonal filter banks and wavelet bases," IEEE Transactions on Signal Processing, vol. 43, no. 3, pp. 649-665, 1995.

[19] May 2010, http://watermarking.unige.ch/Checkmark/index .html. 
[20] S. Voloshynovskiy, S. Pereira, T. Pun, J. J. Eggers, and J. K. $\mathrm{Su}$, "Attacks on digital watermarks: Classification, estimationbased attacks, and benchmarks," IEEE Communications Magazine, vol. 39, no. 8, pp. 118-125, 2001.

[21] May 2010, http://www.ece.uvic.ca/ mdadams/jasper/.

[22] Y. Wang and A. Pearmain, "Blind image data hiding based on self reference," Pattern Recognition Letters, vol. 25, no. 15, pp. 1681-1689, 2004.

[23] E. Elbasi and A. M. Eskicioglu, "A DWT-based robust semiblind image watermarking algorithm using two bands," in Security, Steganography, and Watermarking of Multimedia Contents VIII, vol. 6072 of Proceedings of SPIE, San Jose, Calif, USA, January 2006. 